\title{
High-Temperature Optical Characterization of Wide Band Gap Light Emitting Diodes and Photodiodes for Future Power Module Application
}

Syam Madhusoodhanan ${ }^{1}$, Abbas Sabbar ${ }^{1}$, Sattar Al-Kabi ${ }^{1}$, Stanley Atcitty $^{2}$, Robert Kaplar $^{2}$, Binzhong Dong ${ }^{3}$, Jiangbo Wang ${ }^{3}$, Shui- $^{-}$ Qing $\mathrm{Yu}^{1}$, Zhong Chen ${ }^{*}, 1$

${ }^{1}$ Department of Electrical Engineering, University of Arkansas, Fayetteville, Arkansas 72701, USA

${ }^{2}$ Sandia National Laboratories, Albuquerque, New Mexico 87185, USA

${ }^{3}$ HC SemiTek (Suzhou), 28 Chenfeng Road, Zhangjiagang, Jiangsu, P. R. 215600, China

\author{
A R T I C L E I N F O \\ Article history: \\ Received: 20 December, 2018 \\ Accepted: 22 February, 2019 \\ Online : 06 March, 2019 \\ Keywords: \\ High-Density Power Module, \\ High-Temperature Optocouplers, \\ Light Emitting Diodes, \\ Optical Characterization, \\ Photodiodes, \\ Wide Bandgap
}

\begin{abstract}
A B S T R A C T
A systematic study of wide bandgap (WBG) based light emitting diodes (LEDs) and photodiodes (PDS) were conducted for the assessment of modular integration of optoelectronic devices into power modules. The temperature dependence of the photoluminescence (PL) efficiency of Indium gallium nitride/Gallium nitride (InGaN/GaN) multiple quantum wells (MQWs) material was studied from 10 to $800 \mathrm{~K}$. The photoluminescence efficiency is calculated using the power law relation connecting the integrated photoluminescence signal and the excitation pump power. A peak PL efficiency of $43.97 \%$ was recorded at $800 \mathrm{~K}$. Electroluminescence (EL) study was conducted on Gallium nitride $(G a N)$ based LEDs in the temperature range of $300-800 \mathrm{~K}$. An intensity drop of three orders of magnitude is recorded at $800 \mathrm{~K}$ as compared to room temperature. The full width half maximum (FWHM) of the spectra was also calculated from the EL measurements. The S-shaped shift of FWHM at high temperature indicates a strong localization effect. High-temperature spectral response analysis of $4 \mathrm{H}$-Silicon carbide $4 \mathrm{H}$ $\mathrm{SiC}$ ) pn-junction photodiode is performed at zero voltage bias condition. Enhancement of spectral response is observed at higher wavelengths due to increased phonon population at higher temperatures.
\end{abstract}

\section{Introduction}

This paper is an extension of work originally presented in Conference on Lasers and Electro-Optics (CLEO): Applications and Technology 2018 titled 'Investigation of High-Temperature Photoluminescence Efficiency from InGaN/GaN MQWs' [1]. Power electronic system plays a significant role in renewable energy, energy storage, and electric or hybrid electric vehicles. As an integral part of the power electronic system, power modules provide a highly efficient form of power conversion. In the last two decades, the field of power conversion is going through a transformation where the application often demands extreme operating environments. Advancement in the field of hybrid electric vehicles, aerospace and deep oil-gas exploration necessitate the development of power modules based on WBG devices to operate in extreme environments where the ambient

"Zhong Chen, University of Arkansas, chenz@uark.edu

www.astesj.com

https://dx.doi.org/10.25046/aj040203 temperature exceeds 200oC. Compared to silicon (Si) devices, wide bandgap materials like $\mathrm{SiC}$ and $\mathrm{GaN}$ can withstand higher current densities, exhibit lower switching losses and possess higher breakdown voltages [2-3].

Power modules based on WBG materials enhance reliability and considerably reduce cooling requirements that lead to a significant reduction in total system cost and weight. Although these innovative properties lead power modules to higher power density [4], some concerns still need to be addressed to take full advantage of wide bandgap based modules. For example, the use of bulky transformers used as a galvanic isolation system to float the high voltage gate driver limit further size reduction of the hightemperature power modules [5]. Bulky transformers can be replaced by integrating high-temperature optocouplers to scale down power modules further and achieve disrupting performance 
in terms of thermal management, power efficiency, power density, operating environments, and reliability. However, regular semiconductor optoelectronic materials and devices have major difficulty functioning in high-temperature environments. The motivation for this study is to develop optoelectronic devices, specifically optocouplers that can be integrated into high-density power modules. A detailed study on optoelectronic devices at high temperature enables us to explore the possibility of scaling highdensity power modules by integrating high-temperature optoelectronic devices into the power module.

Modular integration of optoelectronic devices into hightemperature power modules is restricted due to the significant optical efficiency drop at elevated temperatures. The quantum efficiency and long-term reliability of optoelectronic devices decrease at elevated temperatures. Thermal studies show that high junction temperature will significantly lower the lifetime of the LED [6-7]. Performance, as well as the useful life period of the LEDs, will significantly go down under high temperatures. It was also found that high leakage current [8-13], dark spot generation $[10,11]$, and degradation of the metal contacts $[9,12,13]$ at high temperatures can often lead to the degradation of light intensity. These factors limit the application of LEDs in high-temperature environments.

Photodiodes (PDs) based on $\mathrm{SiC}$ has lower leakage current and better stability at high-temperature operation. Brown et al. demonstrated a $6 \mathrm{H}-\mathrm{SiC}$ pn-junction based $\mathrm{PD}$ operation at temperatures up to $350 \mathrm{oC}$ [13]. Several groups have reported $6 \mathrm{H}-$ $\mathrm{SiC}$ pn-junction photodiodes in the past [15-17]. Schottky-barrier PDs are also tested for high-temperature environments in recent years. Different groups have studied and characterized $4 \mathrm{H}-\mathrm{SiC}$ Schottky-barrier and pn-junction PDs at high temperatures [18,19]. Although high quantum efficiency was reported up to $350 \mathrm{oC}$, no groups have ever reported a detailed study on the temperature dependence of the spectral response of $\mathrm{SiC}$ pn-junction photodiodes for a wide range of temperatures. In this paper, we report on the performance degradation of InGaN based LED material and LED devices at high temperatures. Also, a study on the temperature dependence of the response in SiC-based pnjunction PDs up to $527 \mathrm{oC}(800 \mathrm{~K})$ is presented.

\section{Experiment}

High-temperature photoluminescence studies were conducted on the InGaN/GaN MQW structure as described in the sketch of Fig. 1. The InGaN/GaN MQW structures were grown on sapphire substrates. The quantum well structure comprised of 10 periods of $3 \mathrm{~nm}$ undoped $\mathrm{InGaN}$ wells and $12.5 \mathrm{~nm}$ Si-doped GaN barriers grown on a $0.4 \mu \mathrm{m}$ thick n-GaN layer. Vertically structured GaNbased bare die blue LEDs, fabricated at Cree, were subjected to high-temperature electroluminescence studies. The LED structures were grown on $\mathrm{SiC}$ substrate with both contact layers were formed by highly reflective electrodes. Optical responsivity measurements were conducted on $4 \mathrm{H}-\mathrm{SiC}$ pn-junction PDs purchased from ifw Optronics $\mathrm{GmbH}$. The material and device selection for this study are based on the demonstrated high quality, performance, and reliability.

Temperature-dependent measurements on materials and devices were performed using a Janis ST-100 cryostat. The cryostat can reach temperatures up to $800 \mathrm{~K}$. Temperature-

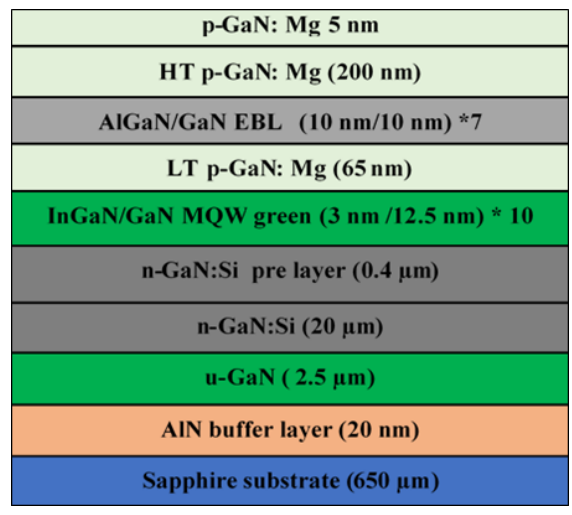

Figure 1. A sketch represents the InGaN/GaN MQWs structure.

dependent PL measurements were carried out using a customary off-axis configuration. The excitation source was a continuous wave laser operating at $395 \mathrm{~nm}$ laser beam diameter of $64 \mu \mathrm{m}$. The power instance of the sample was changed from $1.0-110.0 \mathrm{~mW}$. A Horiba 550 spectrometer combined with a photomultiplier tube is used to collect PL and EL spectra. A Lakeshore 335 temperature controller is used to vary the temperature. Vacuum environment is maintained inside the cryostat throughout the measurements using a Janis TS-75-W turbopump. The injection current during the EL measurements was limited to $1.0 \mathrm{~mA}$ to avoid the device burn out due to the high current density. A Keithley 2450 source measurement unit is used for precise injection of current to the device. Temperature-dependent optical responsivity measurements on 4H-SiC PDs were carried out using Spex 270M rapid scanning imaging monochromator coupled with a deuterium lamp. The wavelength was changed from 250 to $400 \mathrm{~nm}$.

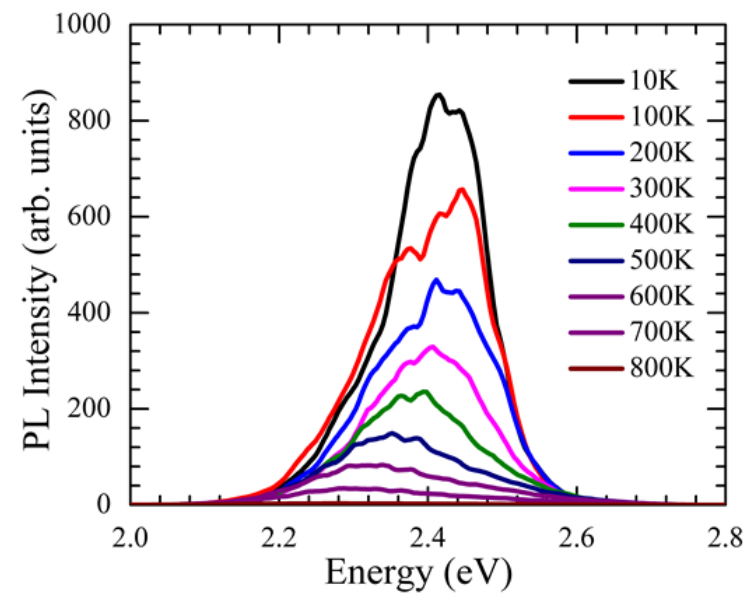

Figure 2. Temperature-dependent photoluminescence spectra of InGaN/GaN MQW were taken in the temperature range of $10-800 \mathrm{~K}$.

\section{Results and Discussion}

The photoluminescence efficiency of the InGaN/GaN MQW structure was measured using the power law relation connecting the integrated PL intensity and the excitation pumping power of the spectrum [20]. Figure 2 shows PL spectra for the InGaN/GaN MQWs for a temperature range from 10 to $800 \mathrm{~K}$. A gradual reduction in the intensity is observed on the high energy side of the PL spectra at elevated temperatures. It is observed from the plot 
that the PL band of InGaN/GaN MQWs become wider at elevated temperatures. A strong Indium (In) segregation can be attributed to the temperature independent behavior of the low energy side [21]. The strong In segregation leads to the formation of In-rich quantum dot-like regions [22]. The spectral peak positions were redshifted from $2.41 \mathrm{eV}$ at $10 \mathrm{~K}$ to $2.32 \mathrm{eV}$ at $800 \mathrm{~K}$. Varshni empirical equation explain the redshift due to the bandgap shrinkage [23].

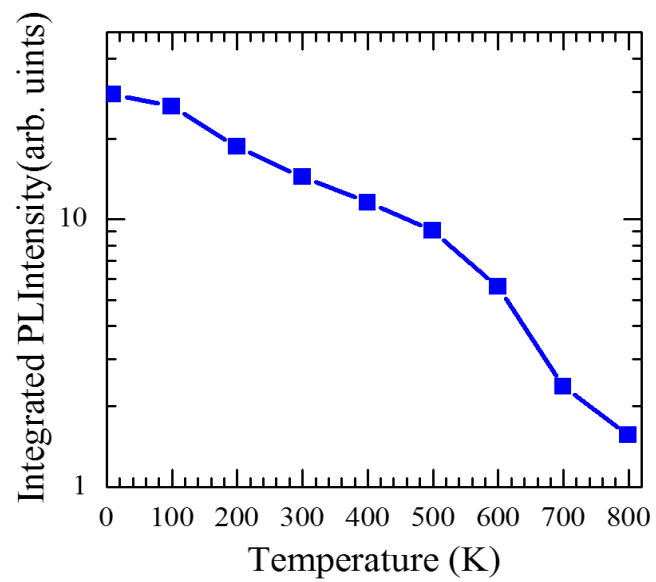

Figure 3. Integrated photoluminescence intensity of $\mathrm{InGaN} / \mathrm{GaN} \mathrm{MQW}$ is plotted as a function of temperature.

Figure 3 shows the integrated PL intensity for the InGaNrelated PL emission for different temperatures. A reduction of one magnitude in the integrated PL intensity at $800 \mathrm{~K}$ compared to 77 $\mathrm{K}$ suggests a high PL efficiency even at elevated temperatures. Domination of non-radiative recombination at higher temperature causes a reduction in PL intensity. Carrier escape at higher temperature due to high thermal energy and the high rate of nonradiative recombination in QWs lead to the intensity drop at high temperature.

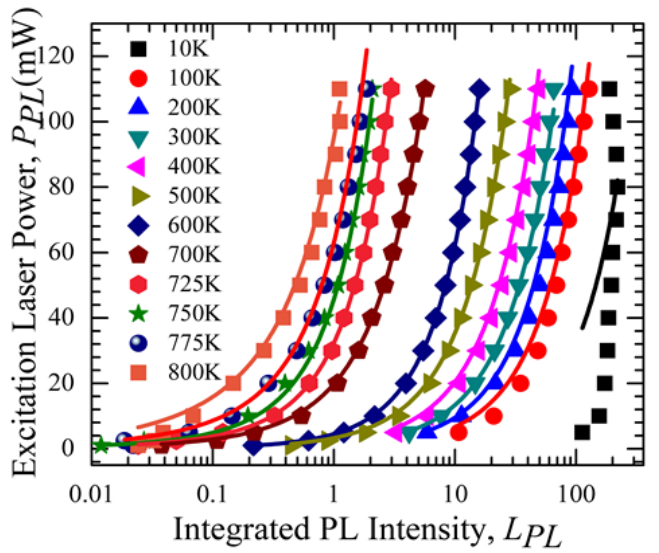

Figure 4. Excitation laser power is plotted against integrated PL intensity from InGaN/GaN MQW at different temperatures. The solid curves are fits of power law model to the data.

The extraction of PL efficiency by the power-law equation is explained in Ref. [19]. Excitation power dependent PL measurements were taken with pump power ranging from $1.0 \mathrm{~mW}$ to $110.0 \mathrm{~mW}$. The results are plotted in terms of excitation pump power as a function of integrated PL intensity in Fig. 4. The dots are the experiment results while the solid curves are the fits of Eq. (1) from Ref. [19] to the data.

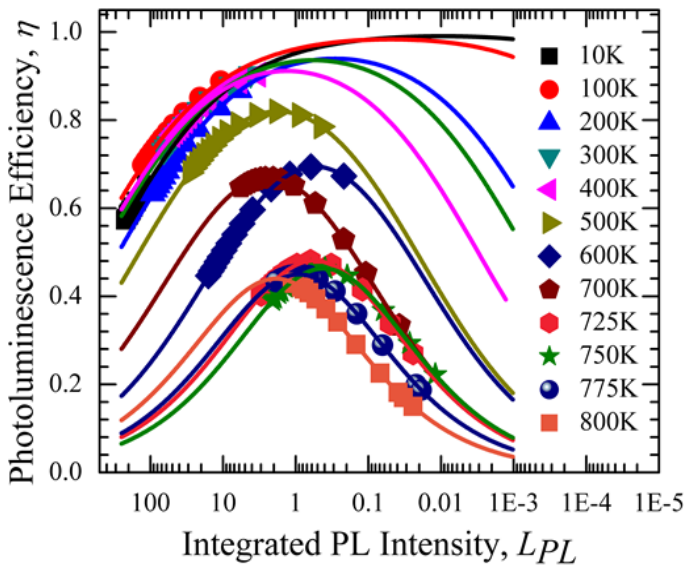

Figure 5. Photoluminescence efficiency is plotted as a function of integrated PL intensity at different temperatures. The solid lines are the simulated data for injection levels above and below the experiment limitations.

Figure (5) shows the temperature-dependent PL efficiency versus integrated PL intensity. The laser injection level where the peak PL efficiency is observed increases as the temperature increases. At $800 \mathrm{~K}$, the peak PL efficiency is observed at an injection level above $110.0 \mathrm{~mW}$. This behavior indicates that the non-radiative recombination rates are dominant even at a low injection rate under room temperature. At elevated temperatures, non-radiative recombinations along with thermally activated carrier escape from the QWs cause the PL efficiency to drop. These results indicate that the Auger and Shockley-Read-Hall (SRH) recombination rates are dependent on temperature and injection level.

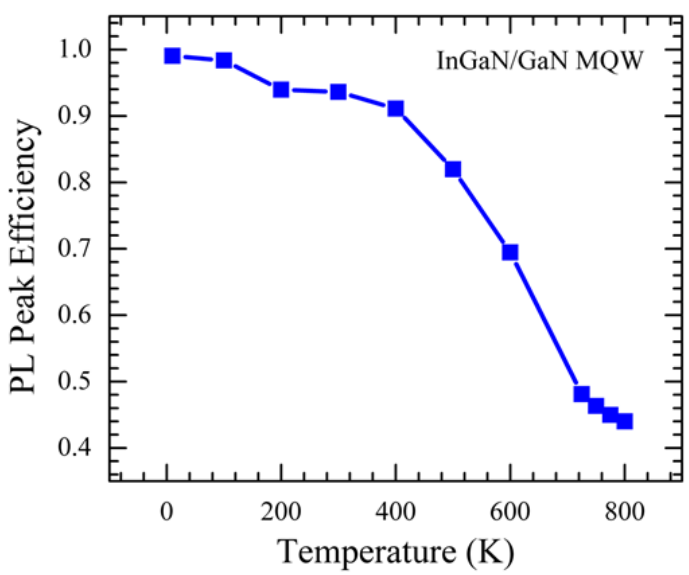

Figure 6. PL peak efficiency of InGaN/GaN MQW is plotted as a function of temperature.

The peak PL efficiency was plotted against temperature as shown in Fig. 6. The peak PL efficiency is almost unity at $10 \mathrm{~K}$. At elevated temperatures, the peak PL efficiency decreases. As mentioned earlier, the drop in the Pl efficiency is caused by the domination of nonradiative recombinations in the active region of the quantum wells. At high temperatures, both SRH and Auger recombinations suppress the radiative recombination resulting in a PL efficiency drop. Another reason for the drop in PL efficiency is due to the carrier escape from the quantum wells as a result of high 
thermal energy. A PL efficiency of $44 \%$ is observed for the sample at $800 \mathrm{~K}$. The temperature independent behavior of PL efficiency up to $400 \mathrm{~K}$ indicates high thermal stability.

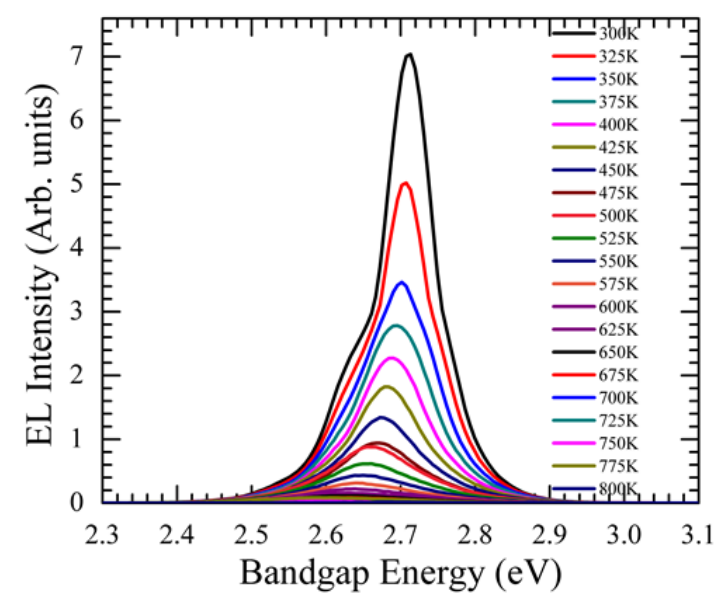

Figure 7. Electroluminescence spectra of InGaN/GaN single quantum well LED measured at different temperatures ranging from $300-800 \mathrm{~K}$.

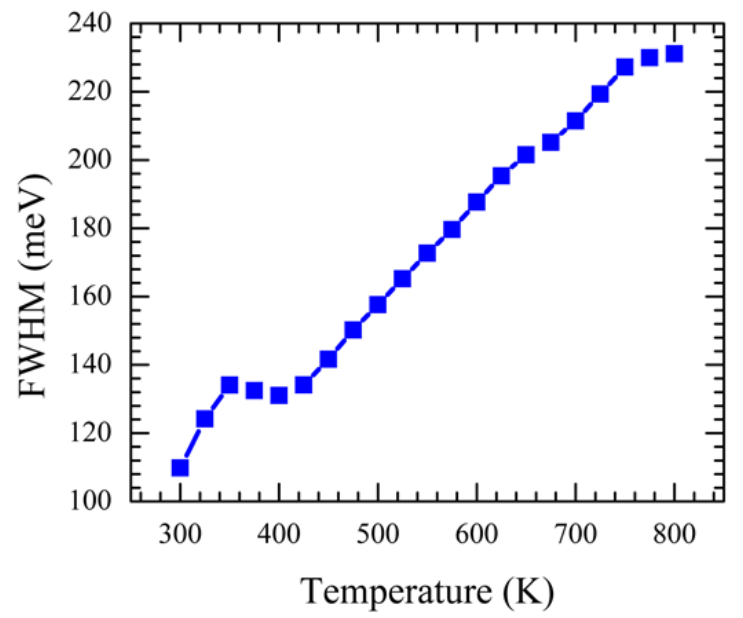

Figure 8. The full width half maximum of the electroluminescence spectra is calculated and plotted against temperature for $\mathrm{InGaN} / \mathrm{GaN}$ single quantum well LED from $300-800 \mathrm{~K}$.

Temperature-dependent electroluminescence spectra of the InGaN/GaN single quantum well (SQW) LED at an injection current of $1 \mathrm{~mA}$ is shown in Fig. 7. The spectral intensity of the LED is decreased as the temperature is increased from 300 to 800 $\mathrm{K}$. The EL peak energy is measured to be $2.71 \mathrm{eV}$ at $300 \mathrm{~K}$. The Indium composition is approximated to be as $16 \%$ from the EL peak energy [24]. The EL peak energy changes from 2.72 to 2.60 $\mathrm{eV}$ as the temperature increased from 300 to $800 \mathrm{~K}$. The EL spectra are fitted using a Gaussian model to calculate the full-width half maximum (FWHM). It is found that the FWHM of the spectra increased from $109.11 \mathrm{meV}$ at $300 \mathrm{~K}$ to $230.11 \mathrm{meV}$ at $800 \mathrm{~K}$. Temperature dependence of the FWHM of the EL spectra for InGaN/GaN LED is shown in Fig. 8. The FWHM shows an Sshaped shift when the temperature increases. The S-shaped shift again indicates a strong localization effect [25].

The FWHM of the spectra increased from $109.11 \mathrm{meV}$ at 300 $\mathrm{K}$ to $230.11 \mathrm{meV}$ at $800 \mathrm{~K}$. The FWHM increases from $300-350$ $\mathrm{K}$ due to carrier relaxation in the localized states. The further increase in the temperature results in a drop in the FWHM until the temperature reaches $400 \mathrm{~K}$. The drop in the FWHM when the temperature further increases to $400 \mathrm{~K}$ suggests that nonradiative recombination centers capture a part of the carriers before reaching the lower energy tail states. The FWHM increases when the temperature increases above $400 \mathrm{~K}$. This monotonic increase in FWHM is caused by the coupling of the excitons to acoustic phonons and longitudinal optical (LO) phonons [26].

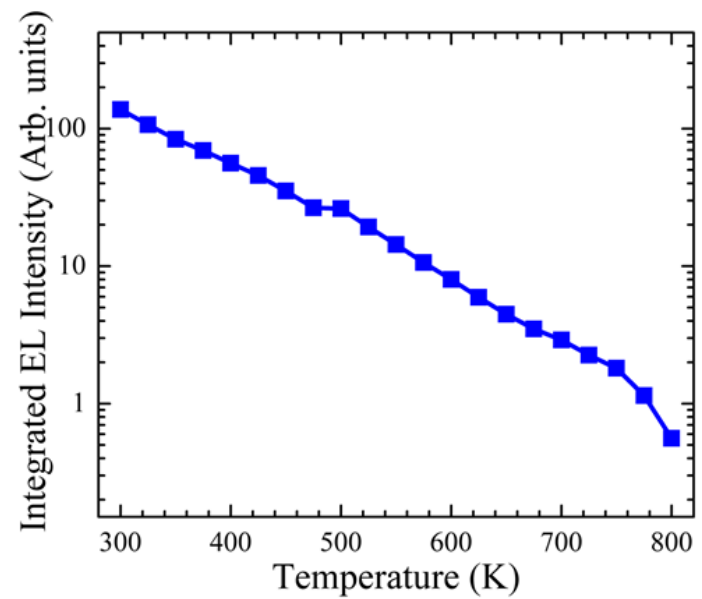

Figure 9. The integrated intensity of the electroluminescence spectra at each temperature is calculated and plotted as a function of temperature for InGaN/GaN single quantum well LED.

Figure 9 shows the change in the integrated intensity of the InGaN/GaN SQW LED over the temperature range of $300-800$ $\mathrm{K}$. The integrated intensity decreases when the temperature increases. The integrated intensity remains the same in the temperature range of $475-500 \mathrm{~K}$. An intensity drop of three orders of magnitude is recorded at $800 \mathrm{~K}$ as compared to room temperature. The three orders of magnitude drop in the intensity suggest the presence of shallow QWs in the InGaN/GaN structure. The reduced intensity at a high temperature is due to the domination of the nonradiative recombination. At elevated temperature, the injected carriers gain high thermal energy. Carrier escape due to high thermal energy leads to the intensity drop at high temperature.

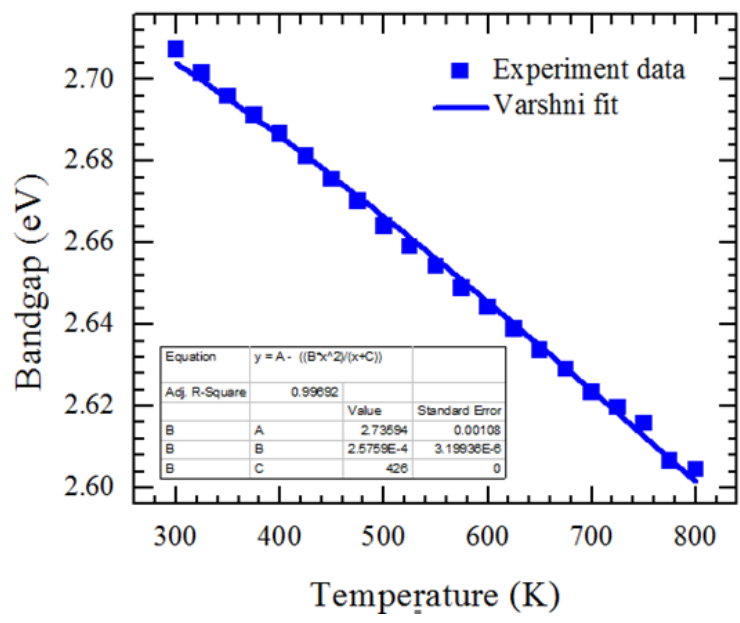

Figure 10. Energy bandgap of InGaN/GaN single quantum well LED is measured and plotted against temperature. 
Figure 10 shows the change in the EL peak energy of the InGaN/GaN SQW LED concerning temperature. The EL peak energy decreases when the temperature increases. The rate of change in EL peak energy remains almost constant during the temperature range of $300-750 \mathrm{~K}$. The EL peak energy changes from 2.71 to $2.60 \mathrm{eV}$ over the temperature range of $300-800 \mathrm{~K}$. The temperature dependence of the EL peak energy is described by the Varshni relation [23].

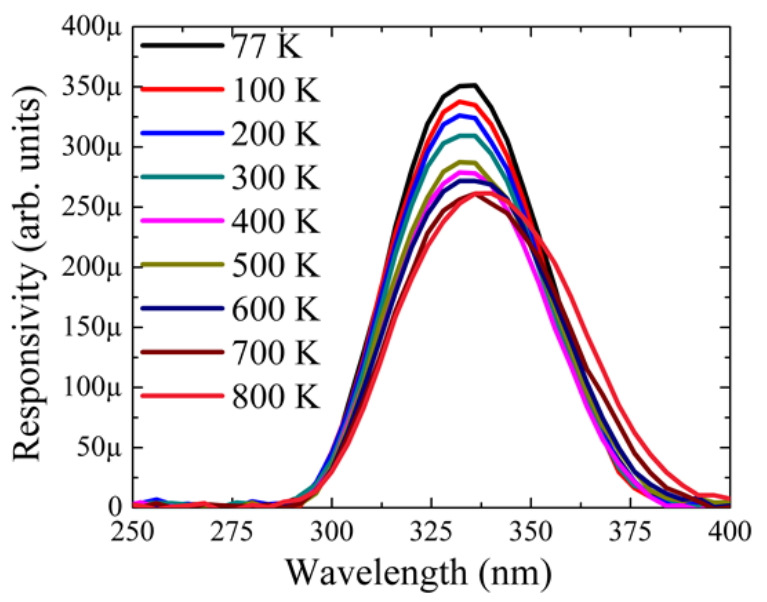

Figure 11. The spectral response characteristics of $4 \mathrm{H}-\mathrm{SiC}$ pn-junction photodiode at different temperatures under $0 \mathrm{~V}$ bias.

Figure 11 shows the spectral response characteristics of the $4 \mathrm{H}-$ $\mathrm{SiC}$ detector measured as a function of temperature from $77 \mathrm{~K}$ to $800 \mathrm{~K}$ at zero biased condition. The photodetectors spectral response is wavelength dependent on the incident light. This is caused by a different energy absorption rate at each wavelength. The spectral response curves shift towards longer wavelength, and the responsivity at a wavelength above $350 \mathrm{~nm}$ is improved at higher temperatures. The redshift is caused by thermally induced bandgap narrowing effect. The observed spectral enhancement at higher wavelengths can be attributed to the increased phonon population at higher temperatures. Phonons assisted photon absorption is necessary at longer wavelengths as the corresponding photon energy is smaller than the direct energy spacing of $4 \mathrm{H}-\mathrm{SiC}$ [19]. Interestingly, the responsivity at a lower wavelength $(<320 \mathrm{~nm})$ exhibits less temperature dependent. The responsivity spectra of the photodiode at lower wavelength merges indicating that at this region temperature has less influence on the responsivity. More studies are necessary to understand the degradation of detectors under high temperature thoroughly.

\section{Conclusion}

Performance degradation of GaN-based LED material, and devices are studied for a wide range of temperature. Spectral responsivity of a $4 \mathrm{H}-\mathrm{SiC}$ pn-junction photodiode is evaluated for the temperature range of $77-800 \mathrm{~K}$. The InGaN/GaN MQW structure shows temperature independent PL efficiency up to 400 $\mathrm{K}$, signifying high thermal stability and displays a peak PL efficiency of $44 \%$ at $800 \mathrm{~K}$. The EL intensity of the LED device was decreased by three orders of magnitude at high temperatures as compared to room temperature. The reduced EL intensity is caused by the carrier escape from the quantum well due to high thermal energy. A strong localization effect is speculated due to the S-shaped FWHM curve. Initial studies on the 4H-SiC pnjunction photodiode show a temperature independent spectral response at lower wavelengths. These results serve as a strong foundation for future studies in the field of high-temperature optoelectronics in the applications of future ultra-high density power modules.

\section{Conflict of Interest}

The authors declare no conflict of interest.

\section{Acknowledgment}

The authors acknowledge financial support from the United States Department of Energy, DOE (Grant No. DE-SC0016485), program manager Dr. Imre Gyuk. Sandia National Laboratories is a multi-program laboratory managed and operated by National Technology Engineering Solutions of Sandia, LLC (NTESS), a wholly owned subsidiary of Honeywell Corporation, for the U.S. Department of Energy's National Nuclear Security Administration under contract DE- NA0003525. The views expressed in this article do not necessarily represent the views of the U.S. Department of Energy or the United States Government.

\section{References}

[1] Sabbar, A., Madhusoodhanan, S., Al-Kabi, S., Dong, B., Wang, J., Yu, S., \& Chen, Z. (2018). Investigation of High-Temperature Photoluminescence Efficiency from InGaN/GaN MQWs. Conference on Lasers and ElectroOptics. doi:10.1364/cleo_at.2018.jth2a.79

[2] Homberger, J., Lostetter, A., Olejniczak, K., Mcnutt, T., Lal, S., \& Mantooth, A. (n.d.). Silicon-carbide ( $\mathrm{SiC}$ ) semiconductor power electronics for extreme high-temperature environments. 2004 IEEE Aerospace Conference Proceedings (IEEE Cat. No.04TH8720). doi:10.1109/aero.2004.1368048

[3] Nomura, T., Masuda, M., Ikeda, N., \& Yoshida, S. (2008). Switching Characteristics of GaN HFETs in a Half Bridge Package for High Temperature Applications. IEEE Transactions on Power Electronics, 23(2), 692-697. doi:10.1109/tpel.2007.915671

[4] Katsis, D. C., \& Zheng, Y. (2008). Development of an extreme temperature range silicon carbide power module for aerospace applications. 2008 IEEE Power Electronics Specialists Conference. doi:10.1109/pesc.2008.4591943

[5] Rowden, B., Mantooth, A., Ang, S., Lostetter, A., Hornberger, J., \& Mcpherson, B. (2009). High Temperature SiC Power Module Packaging. Volume 5: Electronics and Photonics. doi:10.1115/imece2009-12883

[6] Narendran, N., \& Gu, Y. (2005). Life of LED-Based White Light Sources. Journal of Display Technology, 1(1), 167-171. doi:10.1109/jdt.2005.852510

[7] Huang, F., Yin, L., Li, S., Xu, G., Yan, H., Chen, Y., . . Z Zhang, J. (2011). Study on the reliability of high-power LEDs under temperature cycle. 2011 12th International Conference on Electronic Packaging Technology and High Density Packaging. doi:10.1109/icept.2011.6067024

[8] Cao, X., Sandvik, P., Leboeuf, S., \& Arthur, S. (2003). Defect generation in InGaN/GaN light-emitting diodes under forward and reverse electrical stresses. Microelectronics Reliability, 43(12), 1987-1991. doi:10.1016/j.microrel.2003.06.001

[9] Osiński, M., Zeller, J., Chiu, P., Phillips, B. S., \& Barton, D. L. (1996). AlGaN/InGaN/GaN blue light emitting diode degradation under pulsed current stress. Applied Physics Letters, 69(7), 898-900. doi:10.1063/1.116936

[10] Cao, X., Teetsov, J., Shahedipour-Sandvik, F., \& Arthur, S. (2004). Microstructural origin of leakage current in $\mathrm{GaN} / \mathrm{InGaN}$ light-emitting diodes. Journal of Crystal Growth, 264(1-3), 172-177. doi:10.1016/j.jcrysgro.2004.01.031

[11] Egawa, T., Jimbo, T., \& Umeno, M. (1997). Characteristics of InGaN/AlGaN light-emitting diodes on sapphire substrates. Journal of Applied Physics, 82(11), 5816-5821. doi: $10.1063 / 1.366450$

[12] Bulashevich, K. A., Evstratov, I. Y., Mymrin, V. F., \& Karpov, S. Y. (2007) Current spreading and thermal effects in blue LED dice. Physica Status Solidi (c), 4(1), 45-48. doi:10.1002/pssc.200673502

[13] Cao, X., Stokes, E., Sandvik, P., Taskar, N., Kretchmer, J., \& Walker, D. (2002). Optimization of current spreading metal layer for GaN/InGaN-based light emitting diodes. Solid-State Electronics, 46(8), 1235-1239. doi:10.1016/s0038-1101(02)00023-0 
[14] Brown, D., Downey, E., Ghezzo, M., Kretchmer, J., Saia, R., Liu, Y., . . . Schneider, W. (1993). Silicon carbide UV photodiodes. IEEE Transactions on Electron Devices, 40(2), 325-333. doi:10.1109/16.182509

[15] Campbell, R., \& Chang, H. (1966). Detection of ultraviolet radiation using silicon carbide P-N junctions. 1966 International Electron Devices Meeting. doi:10.1109/iedm.1966.187651

[16] Glasow, P., Ziegler, G., Suttrop, W., Pensl, G., \& Helbig, R. (1988). SiC-UVPhotodetectors. Optoelectronic Technologies for Remote Sensing from Space. doi:10.1117/12.943598

[17] Edmond, J., Kong, H., Suvorov, A., Waltz, D., \& Carter, J. C. (1997). 6HSilicon Carbide Light Emitting Diodes and UV Photodiodes. Physica Status Solidi (a), 162(1), 481-491. doi:10.1002/1521-396x(199707)162:13.0.co;2-o

[18] Watanabe, N., Kimoto, T., \& Suda, J. (2012). 4H-SiC pn Photodiodes with Temperature-Independent Photoresponse up to $300 \mathrm{oC}$. Applied Physics Express, 5(9), 094101. doi:10.1143/apex.5.094101

[19] Blank, T. V., Goldberg, Y. A., Kalinina, E. V., Konstantinov, O. V., Konstantinov, A. O., \& Hallén, A. (2005). Temperature dependence of the photoelectric conversion quantum efficiency of $4 \mathrm{H}-\mathrm{SiC}$ Schottky UV photodetectors. Semiconductor Science and Technology, 20(8), 710-715. doi:10.1088/0268-1242/20/8/010

[20] Johnson, S. R., Ding, D., Wang, J., Yu, S., \& Zhang, Y. (2007). Excitation dependent photoluminescence measurements of the nonradiative lifetime and quantum efficiency in GaAs. Journal of Vacuum Science \& Technology B: Microelectronics and Nanometer Structures, 25(3), 1077. doi: $10.1116 / 1.2720864$

[21] Shchekin, O. B., Epler, J. E., Trottier, T. A., Margalith, T., Steigerwald, D. A., Holcomb, M. O. et al Krames, M. R. (2006). High performance thin-film flip-chip InGaN-GaN light-emitting diodes. Applied Physics Letters, 89(7), 071109. doi:10.1063/1.2337007

[22] Bogdanov, M. V., Bulashevich, K. A., Khokhlev, O. V., Evstratov, I. Y., Ramm, M. S., \& Karpov, S. Y. (2010). Current crowding effect on light extraction efficiency of thin-film LEDs. Physica Status Solidi (c), 7(7-8), 2124-2126. doi: $10.1002 /$ pssc. 200983415

[23] Y. P. Varshni, "Temperature dependence of the energy gap in semiconductors," Physica, (Utrecht), no. 34, pp. 149 -154, 1967, http://dx.doi.org/10.1016/0031-8914(67)90062-6

[24] Wu, J., Walukiewicz, W., Yu, K. M., Ager, J. W., Haller, E. E., Lu, H., \& Schaff, W. J. (2002). Small band gap bowing in In1-xGaxN alloys. Applied Physics Letters, 80(25), 4741-4743. doi:10.1063/1.1489481

[25] Lu, T., Ma, Z., Du, C., Fang, Y., Wu, H., Jiang, Y., . . . Chen, H. "Temperature-dependent photoluminescence in light-emitting diodes, Scientific Reports, 2014, http://dx.doi.org/10.1038/srep06131

[26] Franssen, G., Litwin-Staszewska, E., Piotrzkowski, R., Suski, T., \& Perlin, P. (2003). Optical and electrical properties of homoepitaxially grown multiquantum well InGaN/GaN light-emitting diodes. Journal of Applied Physics, 94(9), 6122-6128. doi:10.1063/1.1616637 